\title{
ARTRODESIS C1C2 CON TORNILLOS TRANSARTICULARES EN ARTRITIS REUMATOIDEA: EXPERIENCIA Y REVISIÓN DE LA LITERATURA
}

\author{
ARTRODESE C1 C2 COM PARAFUSOS TRANSARTICULARES EM ARTRITE REUMATOIDE: \\ EXPERIÊNCIA E REVISÃO DE LITERATURA
}

C1 C2 ARTHRODESIS WITH TRANSARTICULAR SCREWS IN RHEUMATOID ARTHRITIS: EXPERIENCE AND LITERATURE REVIEW

Lyonel Beaulieu Lalanne ${ }^{1}$, Gonzalo Arriagada Ocampo ${ }^{2}$

\begin{abstract}
RESUMEN
Objetivo: Describir los resultados clínicos e imagenológicos utilizando la técnica de fijación C1 C2 con tornillos transarticulares y asas de alambre en pacientes portadores de AR en un seguimiento a largo plazo y revisar la literatura actual. Método: Entre los años 2002 y 2006 , 11 pacientes (9 mujeres y 2 hombres) con inestabilidad C1 C2 secundaria a AR fueron intervenidos quirúrgicamente. Se realizó fijación C1 C2 con tornillos transarticulares por vía posterior más asas de alambre y aplicación de injerto óseo autólogo de cresta ilíaca. Se registró Índice de Ranawat pre y posoperatorio, Distancia Anterior Atlas Odontoides (DAAO) pre y posoperatorio, tiempo operatorio, días de hospitalización, complicaciones intra y posoperatorias y tiempo de consolidación radiológica, con un seguimiento promedio de 34 meses. Resultados: Todos los pacientes presentaron mejoría del Índice de Ranawat en el postoperatorio. La DAAO preoperatoria promedio fue de 11,9 mm (DS $\pm 2,57$ ), rango 7 a 16, y la DAAO postoperatoria promedio fue de $3 \mathrm{~mm}$ (DS $\pm 1,20)$, rango 2 a 6 . El tiempo quirúrgico fue de 94 minutos en promedio y el promedio de días de hospitalización fue de 7 días. No se presentaron complicaciones intraoperatorias. Un caso presentó seroma de herida operatoria que requirió tratamiento quirúrgico. El tiempo de consolidación fue en promedio 14 semanas. Conclusión: La artrodesis atlantoaxial con tornillos y amarras es una buena alternativa para el manejo de la inestabilidad C1-C2 en pacientes portadores de AR, consiguiendo buenos resultados clínicos e imagenológicos en un seguimiento a largo plazo.
\end{abstract}

Descriptores: Artritis reumatoidea; Tornillos óseos; Artrodesis.

\section{RESUMO}

Objetivo: Apresentar a experiência com a técnica de fixação C1-C2 com parafusos transarticulares e cerclagem de fio metálico nos pacientes portadores de AR, assim como a revisão da literatura. Método: Entre os anos 2002 e 2006, 11 pacientes (9 mulheres e 2 homens) com instabilidade C1-C2 e portadores de AR foram submetidos a tratamento cirúrgico. Foi realizada a fixação C1-C2 com parafusos transarticulares por via posterior e cerclagem com fio metálico e enxerto autólogo. O Índice de Ranawat pré e pós-operatorio foi registrado, assim como a distância anterior atlas-dontoide (DAAO), o tempo operatório, os dias de hospitalização, as complicações trans e pósoperatórias e o tempo de consolidação. O seguimento médio foi de 34 meses. Resultados: Todos os pacientes apresentaram melhora do Índice de Ranawat no pós-operatório, a DAAO pré-operatória média foi de 11,9 cm (DP $\pm 2,57$ ), variação de 7 a 16, e a DAAO pósoperatória média foi de $3 \mathrm{~cm}(\mathrm{DP} \pm 1,20)$, com a variação de 2 a 6 . O tempo cirúrgico médio foi de 94 minutos e o período médio de de hospitalização foi 7 dias. Não foram observadas complicações intraoperatórias. Um paciente apresentou seroma de ferida operatória que necessitou de tratamento cirúrgico. O tempo de consolidação foi em média 14 semanas. Conclusão: A artrodese atlanto-axial com parafusos e cerclagem com fio metálico é uma boa alternativa no tratamento da instabilidade C1-C2 nos pacientes portadores de AR, proporcionando bons resultados clínicos e radiológicos.

Descritores: Artrite reumatóide; Parafusos ósseos; Artrodese.

\begin{abstract}
Objective: Present the long term clinical and radiological results in C1-C2 transarticular screws technique with posterior wiring in RA patients and presenting the current literature review. Methods: From 2002 to 2006, eleven patients (9 women and 2 men) with RA and C1-C2 instability underwent $\mathrm{C} 1-\mathrm{C} 2$ arthrodesis with transarticular screws plus posterior wiring and autologous iliac crest bone graft. A retrospective chart and radiographic review was performed. Ranawat Index was recorded in pre- and post-operative, as well as anterior atlantodental interval (AADI), operating time, days of hospitalization and complications. The mean follow up (FU) was 34 months. Results: All patients presented improved Ranawat Index at follow up. Average pre-operative AADI was 11,9mm (SD $\pm 2,57$ ), range 7 to 16. Average post-operative AADI was $3 \mathrm{~mm}$ $(S D \pm 1.20)$, range 2 to 6 . The average operating time was 94 minutes and the average days of hospitalization was 7 days. No intra-operative complications were found. Post-operative seroma was found in one case and surgical drainage was required. All patients achieved solid fusion at 14 weeks in average. Conclusions: C1-C2 arthrodesis with transarticular screws plus posterior wiring and autologous bone graft is a good alternative for atlantoaxial instability due $R A$, with good clinical and radiographic outcomes.
\end{abstract}

Keywords: Rheumatoid Arthritis; Bone screws; Arthrodesis.

1. Miembro Centro de Columna Clínica Las Condes de Santiago, Chile.

2. Miembro Equipo de Columna Instituto Traumatológico de Santiago Dr. Teodoro Gebauer Weisser. Santiago, Chile.

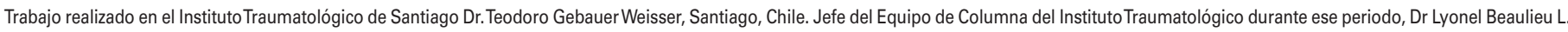
Correspondência: Lo Fontecilla 441, Las Condes. Santiago. Chile. E-mail: Ibeaulieu@ @linicalascondes.cl

Recebido em 17/05/2011, aceito em 09/09/2011 


\section{INTRODUCCIÓN}

En Artritis Reumatoidea (AR), el compromiso de la columna cervical es muy frecuente, algunos artículos muestran frecuencias de hasta el 86\% ${ }^{1}$. La inestabilidad C1 C2 es el más común de estos compromisos presentándose hasta en 2/3 de los $\operatorname{casos}^{2,3}$. La complicación neurológica secundaria al compromiso AR de la columna cervical es la mielopatía cervical

Los factores que se relacionan a mayor riesgo de inestabilidad C1 C2 habitualmente descritos son: sexo masculino, factor reumatoideo positivo, enfermedad articular periférica erosiva severa, uso de corticoides por largo periodo y la presencia de nódulos subcutáneos reumatoideos ${ }^{4}$. El óptimo manejo quirúrgico de la inestabilidad C1 C2 requiere de una artrodesis estable. Existen varias técnicas quirúrgicas para realizar la fijación de este segmento y se han realizado análisis de los factores favorables y desfavorables a cada una de éstas ${ }^{5}$. La técnica de fijación con tornillos transarticulares C1 C2 por vía posterior (Técnica de Magerl) asociados a asas de alambre (Técnica de Brooks), presentan ventajas biomecánicas sobre otros métodos, lo que permitiría prescindir incluso del uso de inmovilización externa post operatoria como el halo chaqueta ${ }^{6,7}$.

Los criterios quirúrgicos para artrodesis C1 C2 en AR son: inestabilidad severa, dolor intratable, signos clínicos y/o imagenológicos de mielopatía cervical, diámetro del canal raquídeo menor de 6 $\mathrm{mm}$ en posición neutra o flexión, Distancia Posterior Odontoides Atlas (DPOA) menor a $14 \mathrm{~mm}$, Distancia Anterior Atlas Odontoides (DAAO) mayor a $9 \mathrm{~mm}$ y diámetro del cordón medular medido por RM menor de $6 \mathrm{~mm}$ en el segmento ${ }^{4,8}$.

El objetivo del presente artículo es mostrar la experiencia con la técnica de fijación C1 C2 con tornillos transarticulares y asas de alambre en AR, y revisar la literatura existente con relación a esta patología y sus alternativas quirúrgicas.

\section{PACIENTES Y MÉTODOS}

Entre enero de 2002 y diciembre de 2006, 11 pacientes con inestabilidad C1 C2 que cumplían criterios de cirugía fueron operados. Se realizó fijación C1 C2 con tornillos transarticulares por vía posterior más asas de alambre y aplicación de injerto óseo autólogo de cresta ilíaca.

La muestra está conformada por 11 pacientes, 9 mujeres y 2 hombres, con una edad promedio de 55 años (rango: 16 a 69 años).

Todos los pacientes se encontraban con terapia farmacológica para AR y habían sido sometidos a otras cirugías en relación a AR, como artroplastía total de caderas y/o rodillas, mano y pie reumatoide.

En todos los casos se realizaron radiografías de columna cervical en proyecciones AP y lateral estándar, C1 C2 transoral (Figura 1) y proyecciones laterales dinámicas (Figura 2), además de Tomografía Computarizada Multicorte y Resonancia Magnética Columna Cervical.

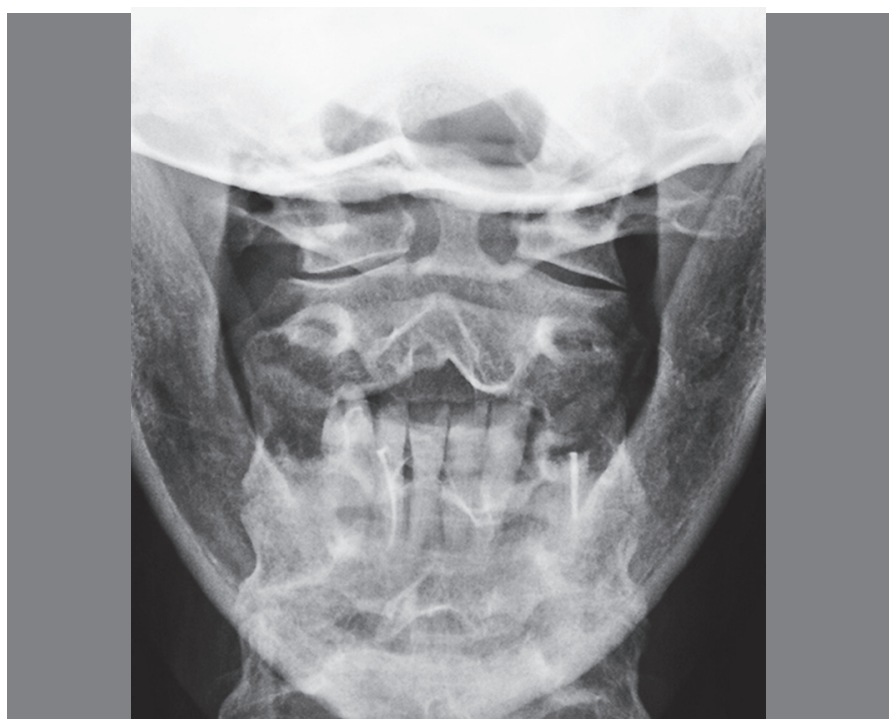

Figura 1. $\mathrm{RX}-\mathrm{C} 1 \mathrm{C} 2$ transoral.
El primer día post operatorio se realizó en la Unidad de Cuidados Intensivos (UCl) y la bipedestación se realizó a las 72 horas. Todos los pacientes se inmovilizaron con collar Filadelfia hasta la fusión radiológica. El seguimiento se realizó con radiografías de columna cervical lateral y C1 C2 transoral a las 48 horas, 3, 6, 9 y 12 semanas, y a los 6,9 y 12 meses post cirugía y control una vez por año posteriormente. Se registró la DAAO e Indice de Ranawat (Tabla 1) preoperatorio y al término del seguimiento. El promedio de seguimiento fue de 34 meses, con un rango de 12 a 66 meses.

\section{Técnica quirúrgica}

La técnica quirúrgica consiste en posicionamiento decúbito prono con compás de Mayfield y control radiológico de la posición. El abordaje quirúrgico fue desde la línea occipital inferior hasta C5, de tal modo de lograr un ángulo adecuado para el posicionamiento de los tornillos (Figura 3). Se identificaron facetas C1 C2 y C2 C3. Se posicionó el injerto autólogo de cresta ilíaca y se fijó con asas de alambre según técnica de Brooks, controlando la adecuada reducción del desplazamiento C1 C2. Bajo control fluoroscópico se pasa una broca guía desde el aspecto inferior y posterior de la carilla articular de C2 en la unión con la lámina dirigido hacia el aspecto posterior del proceso articular superior con un eje a medial de 5 a $10^{\circ}$, para luego penetrar por las facetas a las masas laterales de C1. Luego se coloca un tornillo de $3.5 \mathrm{~mm}$ de diámetro no canulado (Figura 4 y figura 5).

\section{RESULTADOS}

No hubo complicaciones intraoperatorias ni complicaciones en relación al posicionamiento del implante.

El promedio de duración de cirugía fue de 94 minutos y el promedio de días de hospitalización fue de 7 días.

En la evolución tardía 2 pacientes presentaron mareos y 4 pacientes evolucionaron con dolor cráneo cervical, síntomas que remitieron espontáneamente durante el primer mes post operatorio en todos los casos. Un paciente presentó seroma del sitio operatorio que requirió drenaje quirúrgico. Ninguno de los pacientes presentó deterioro de la condición neurológica. La DAAO preoperatoria promedio fue de $11,9 \mathrm{~mm}$ (DS $\pm 2,57$ ), rango 7 a 16 , y la DAAO postoperatoria promedio fue de $3 \mathrm{~mm}$ (DS $\pm 1,20$ ), rango 2 a 6 .

Todos los pacientes mostraron una disminución de la DAAO y mejoría del Indice de Ranawat (Tabla 2).

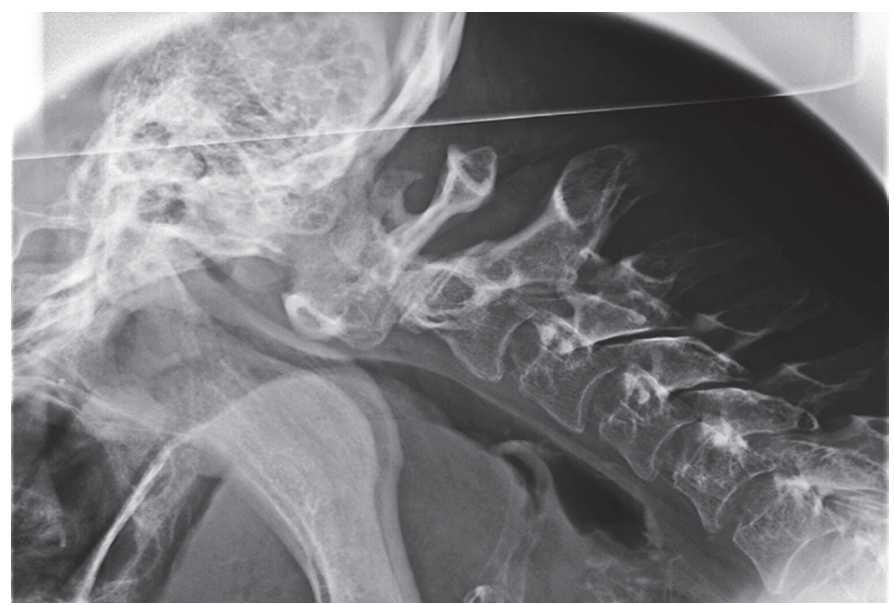

Figura 2. RX - lateral en flexión máxima.

Tabla 1. Clasificación de Ranawat.

\begin{tabular}{c|c}
\hline Tipo & Características Clínicas \\
\hline$I$ & Sin Déficit Neurológico \\
\hline$\|$ & Debilidad subjetiva con hiperreflexia y disestesias \\
\hline$I \|$ A & Debilidad objetivable y "long tract" en pacientes ambulatorios \\
\hline III B & Debilidad objetivable y "long tract" en pacientes no ambulatorios \\
\hline
\end{tabular}



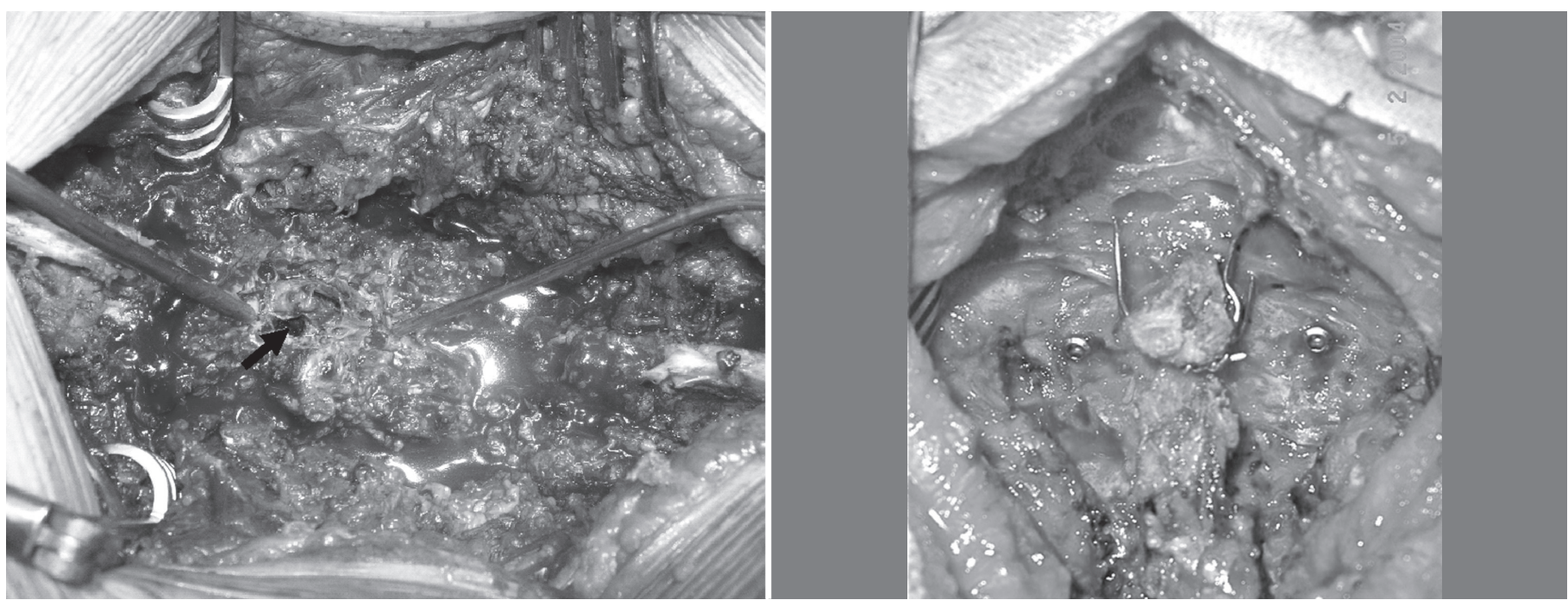

Figura 3. Visión intraoperatoria.

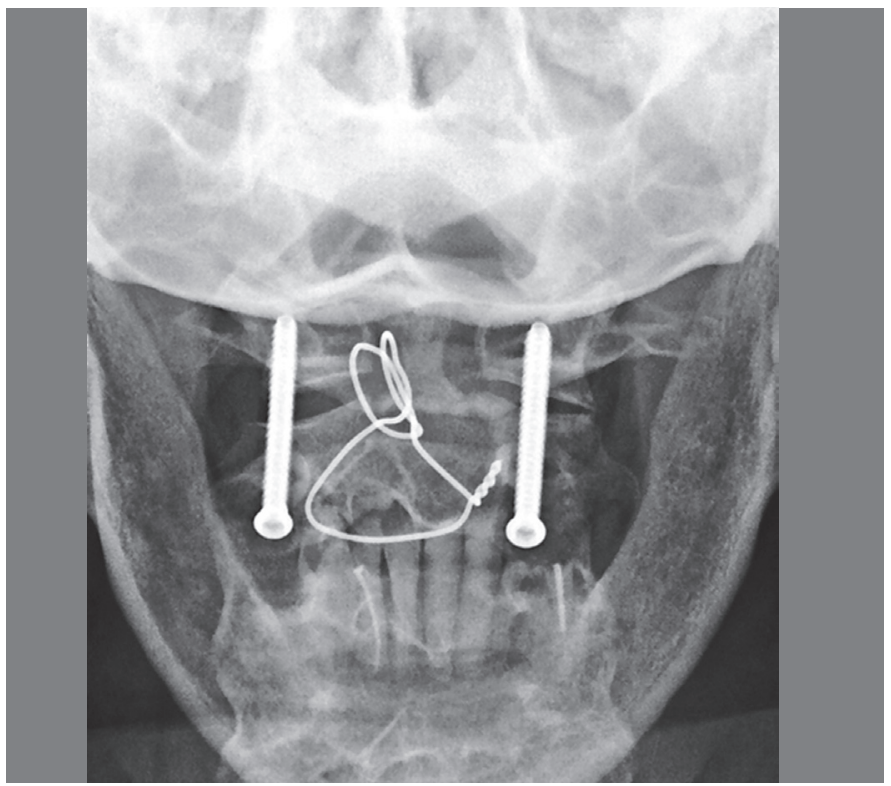

Figura 4. RX - C1 C2 transoral post operatoria.

Tabla 2. Resultados imagenológicos y clínicos.

\begin{tabular}{|c|c|c|c|c|}
\hline Caso & Pre - op & Pre - op & Post - op & Post - op \\
\hline & DAAO $(\mathrm{mm})$ & Indice de Ranawat & DAAO $(\mathrm{mm})$ & $\begin{array}{l}\text { Indice de } \\
\text { Ranawat }\end{array}$ \\
\hline 1 & 7 & II & 2 & । \\
\hline 2 & 15 & III A & 6 & I \\
\hline 3 & 12 & III A & 4 & 1 \\
\hline 4 & 10 & III A & 2 & । \\
\hline 5 & 11 & II & 3 & 1 \\
\hline 6 & 10 & III A & 2 & 1 \\
\hline 7 & 13 & II & 3 & I \\
\hline 8 & 16 & III A & 3 & II \\
\hline 9 & 10 & III A & 2 & 1 \\
\hline 10 & 12 & II & 2 & 1 \\
\hline 11 & 15 & III A & 4 & II \\
\hline
\end{tabular}

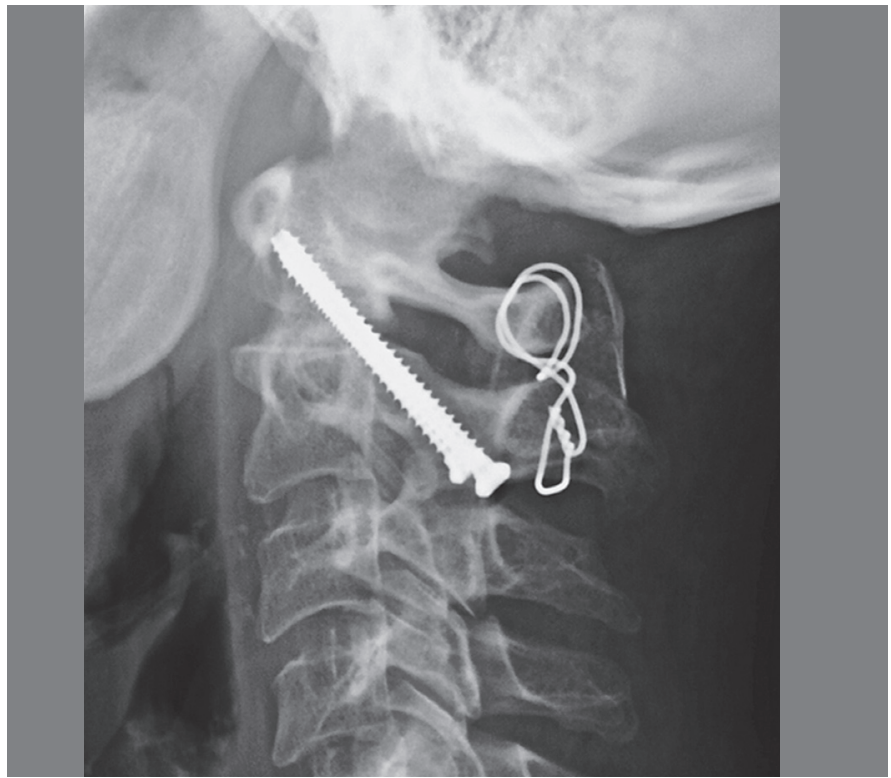

Figura 5. RX - lateral post operatoria.

Dos casos mostraron signos de aflojamiento de las asas de alambre.

A las 14 semanas todos los pacientes presentaron signos radiológicos de fusión.

\section{DISCUSIÓN}

El método tradicional de artrodesis atlanto-axial con amarras sublaminares e injerto óseo autólogo presenta porcentajes de no unión de hasta un $30 \%{ }^{9,10}$. Las características de la artrodesis con tornillos transarticulares C1 C2 permiten mejorar la calidad de la artrodesis atlanto-axoídea, asegurar una estabilización inmediata y adecuada del segmento C1 C2, evitar el daño medular por compresión y lograr un postoperatorio sin requerir el uso de inmovilización rígida externa ${ }^{11}$.

Todos los pacientes operados en esta serie presentaron una mejoría clínica post operatoria, la mayoría dentro del grado I de la clasificación de Ranawat (Tabla 2). Esto es concordante con otras series publicadas $^{6,12}$. Juega un papel importante en esta evolución neurológica favorable la adecuada selección de los pacientes, descartando la cirugía en los pacientes que se encuentran en etapa III B de Ranawat, ya que estos tendrían muy escasas posibilidades de mejoría ${ }^{8,13}$. 
Dentro de esta serie hubo dos pacientes con falla en las amarras sublaminares, pero la reducción se mantuvo hasta la fusión definitva C1 C2. Esto se debería a que, una de las principales características biomecánicas de esta técnica combinada con tornillos transarticulares y amarras sublaminares, es la estabilidad traslacional y rotacional, mejorando las condiciones para la fusión ${ }^{10,14,15}$, llegando incluso al $100 \%$ de artrodesis en diferentes series ${ }^{7,9,16}$, siendo solo necesario el uso de un collar Filadelfia post operatorio $6,7,9$.

Sin embargo, esta técnica es más demandante que el uso exclusivo de amarras, por lo que hay una serie de riesgos como mal posición de los tornillos, rotura dural, lesión de las arterias vertebrales, pseudoartrosis y otras, además de las propias de cualquier acto quirúrgico ${ }^{17}$. La complicación más destacada dentro de la literatura es la lesión de la arteria vertebral, con incidencias descritas variables $7,16,18$ pero que Wright describió en su estudio retrospectivo de un $2.4 \%$ en forma global ${ }^{19}$. No obstante, existen publicaciones en que se presentaron lesiones intraoperatorias de la arteria vertebral pero sin secuelas gracias a la gran circulación colateral del polígono de Willis ${ }^{10}$

Para evitar la malposición de los tornillos transarticulares y sus posibles complicaciones, Dickman y Sonntag plantearon considerar $^{9}$ evitar este procedimiento si la arteria vertebral presenta un recorrido tortuoso o anómalo, por lo que se hace necesario realizar TAC y RM de la zona ${ }^{20}$, tal como se realizó a los pacientes de esta serie. Segundo, tampoco realizar este procedimiento si las masas laterales de C1 o la pars interarticularis de C2 se encuentran dañadas $^{10}$. Se describe que entre un 18 a 23\% de los casos presentarían variaciones anatómicas que no permitirían la utilización de los tornillos transarticulares, por lo que el estudio preoperatorio es de vital importancia para detectar estos casos y plantear otra posibilidad quirúrgica ${ }^{21-23}$. Tercero, el uso de asistencia de navegación puede evitar la malposición, siempre y cuando el equipo quirúrgico esté lo suficientemente familiarizado con esta técnica. Esto no reemplaza el conocimiento acabado de la anatomía y puntos de reparo de este segmento anatómico. Cuarto, el uso de fluoroscopía intra operatoria disminuye ostensiblemente los riesgos de malposición de la osteosíntesis. Quinto, se requiere una adecuada visualización de las pars interarticularis de C2 y de la faceta de C1 C2 durante el acto quirúrgico para obtener unos adecuados puntos de reparo y así una correcta posición de los tornillos transarticulares. Con estas condiciones se puede lograr hasta un 95\% de éxito en la colocación de los tornillos transarticulares ${ }^{24}$

\section{REFERENCIAS}

1. Nguyen HV, Ludwig SC, Silber J, Gelb DE, Anderson PA, Frank L, et al. Rheumatoid arthritis of the cervical spine. Spine J. 2004:4(3):329-34.

2. Dreyer SJ, Boden SD. Natural history of rheumatoid arthritis of the cervical spine. Clin Orthop Relat Res. 1999;(366):98-106.

3. Boden SD, Dodge LD, Bohlman HH, Rechtine GR. Rheumatoid arthritis of the cervical spine. A longterm analysis with predictors of paralysis and recovery. J Bone Joint Surg Am. 1993:75(9):1282-97.

4. Beaulieu L, Vial S, Delgado, San Martín A. Artritis Reumatoídea en Columna Cervical: Algoritmos de Tratamiento. Coluna/Columna. 2005; 4(1):42-19.

5. Monsey RD. Rheumatoid Arthritis of the Cervical Spine. J Am Acad Orthop Surg 1997;5(5):240-248

6. Eleraky MA, Masferrer R, Sonntag VK. Posterior atlantoaxial facet screw fixation in rheumatoid arthritis. J Neurosurg. 1998:89(1):8-12.

7. Grob D, Jeanneret B, Aebi M, Markwalder TM. Atlanto-axial fusion with transarticular screw fixation. J Bone Joint Surg Br. 1991;73(6):972-6

8. Casey AT, Crockard HA, Pringle J, O'Brien MF, Stevens JM. Rheumatoid artritis of the cervical spine: current techniques for management. Orthop Clin North Am. 2002;33(2):291-309.

9. Dickman CA, Sonntag VK. Posterior C1-C2 transarticular screw fixation for atlantoaxial arthrodesis. Neurosurgery. 1998;43(2):275-80.

10. Farey ID, Nadkarni S, Smith N. Modified Gallie technique versus transarticular screw fixation in C1-C2 fusion. Clin Orthop Relat Res. 1999:(359):126-35.

11. Naderi S, Crawford NR, Song GS, Sonntag VK, Dickman CA. Biomechanical comparison of C1-C2 posterior fixations. Cable, graft, and screw combinations. Spine (Phila Pa 1976). $1998 ; 23(18): 1946-55$

12. Nagaria J, Kelleher MO, McEvoy L, Edwards R, Kamel MH, Bolger C. C1-C2 transarticular screw fixation for atlantoaxial instability due to rheumatoid arthritis: a seven-year analysis of outcome. Spine (Phila Pa 1976). 2009;34(26):2880-5

13. Grob D, Schütz U, Plötz G. Occipitocervical fusion in patients with rheumatoid arthritis Clin Orthop Relat Res. 1999:(366):46-53.

14. Puttlitz CM, Melcher RP Kleinstueck FS, Harms J Bradford DS, Lotz JC Stability analysis of craniovertebral junction fixation techniques. J Bone Joint Surg Am. 2004;86(3):561-8.

15. Hanson PB, Montesano PX, Sharkey NA, Rauschning W. Anatomic and biomechanical assessment of transarticular screw fixation for atlantoaxial instability. Spine (Phila Pa 1976). 1991;16(10):1141-5.
También es de vital importancia para evitar complicaciones con la posición de los tornillos una adecuada reducción previa a la colocación de estos, lo que en este serie se realizó colocando las asas de alambre sublaminares antes de los tornillos transarticulares. Madawi et $a^{25}$ publicó una serie en que todos los casos de mal posición y lesiones de las arterias vertebrales se presentaron en pacientes en que no se logró una adecuada reducción previa a la osteosíntesis.

Muchas publicaciones consideran imprescindible una evaluación postoperatoria inmediata con una TC del segmento C1 C2 para determinar la posición de los tornillos transarticulares y su punto de entrada ${ }^{17,26}$, pero en la mayoría de estas series, cuando se detecta un tornillo mal posicionado, no modifica la conducta y se mantiene al paciente en observación, sin reintervención, dado que estos se mantenían asintomáticos

Existe la creencia de que la artrodesis C1 C2 en pacientes AR conlleva a una subluxación y pérdida de la cifosis subaxial, pero se ha demostrado que la alineación de la columna cervical baja depende de varios factores que no sólo incluyen al ángulo de fijación C1 C2, sino que también al desarrollo y/o progresión de lesiones subaxiales y a la disrrupción de los músculos extensores; por lo que actualmente solo se recomienda no realizar la fusión en excesiva lordosis o cifosis ${ }^{27}$.

Se han descrito otras técnicas para una artrodesis C1 C2 en pacientes AR, siendo una de ellas la descrita por Harms de tornillos a las masas laterales de C1 y pars interarticularis de C2. Teóricamente tendría la ventaja de disminuir las posibilidades de complicaciones en relación a la arteria vertebral con la inserción de los tornillos transarticulares, pero de todas maneras el riesgo existe ${ }^{21}$. Asimismo, los resultados biomecánicos de los tornillos transarticulares son similares a los tornillos según la técnica de Harms ${ }^{14} \mathrm{o}$ incluso superiores ${ }^{28}$, ya sea para una fusión C1 C2 como para una fusión occipitocervical (CO C2) $)^{13,14}$

\section{CONCLUSIÓN}

La artrodesis atlanto-axial con tornillos y amarras es una buena alternativa para el manejo de la inestabilidad C1 C2 en pacientes $\mathrm{AR}$, evaluando paciente y cirujano los beneficios y riesgos de esta técnica, así como criterios de selección adecuados. Según la experiencia obtenida, impresiona que el porcentaje de reabsorción del injerto y falla del montaje es menor con esta técnica en relación a la artrodesis con amarras sublaminares, consiguiendo buenos resultados clínicos en los pacientes sometidos a esta intervención.

16. Stillerman CB, Wilson JA. Atlanto-axial stabilization with posterior transarticular screw fixation: technical description and report of 22 cases. Neurosurgery. 1993:32(6):948-54

17. Fountas KN Smisson HF 3rd Robinson JS Jr. C1-C2 transarticular screw fixation for atlantoaxial instability: a 6-year experience. Neurosurgery. 2002;50(3):672-3.

18. Coric D, Branch CL Jr, Wilson JA, Robinson JC. Arteriovenous fistula as a complication of C1-2 transarticular screw fixation. Case report and review of the literature. J Neurosurg. 1996:85(2):340-3

19. Wright NM, Lauryssen C. Vertebral artery injury in C1-2 transarticular screw fixation: results of a survey of the AANS/CNS section on disorders of the spine and peripheral nerves. American Association of Neurological Surgeons/Congress of Neurological Surgeons. J Neurosurg. 1998:88(4):634-40.

20. Dull ST, Toselli RM. Preoperative oblique axial computed tomographic imaging for $\mathrm{C} 1-\mathrm{C} 2$ transarticular screw fixation: technical note. Neurosurgery. 1995;37(1):150-1.

21. Harms J, Melcher RP. Posterior C1-C2 fusion with polyaxial screw and rod fixation. Spine (Phila Pa 1976). 2001;26(22):2467-71.

22. Paramore CG, Dickman CA, Sonntag VK. The anatomical suitability of the C1-2 complex for transarticular screw fixation. J Neurosurg. 1996;85(2):221-4.

23. Resnick DK, Lapsiwala S, Trost GR. Anatomic suitability of the C1-C2 complex for pedicle screw fixation. Spine (Phila Pa 1976). 2002;27(14):1494-8.

24. Bloch O, Holly LT, Park J, Obasi C, Kim K, Johnson JP. Effect of frameless stereotaxy on the accuracy of C1-2 transarticular screw placement. J Neurosurg. 2001:95(1 Suppl):74-9.

25. Madawi AA Casey AT Solanki GA Tuite G. Veres R, Crockard HA. Radiological and anatomical evaluation of the atlantoaxial transarticular screw fixation technique. J Neurosurg. 1997;86(6):961-8.

26. Jun BY. Anatomic study for ideal and safe posterior C1-C2 transarticular screw fixation Spine (Phila Pa 1976). 1998;23(15):1703-7.

27. Mukai Y, Hosono N, Sakaura H, Fujii R, Iwasaki M, Fuchiya T, et al. Sagittal alignment of the subaxial cervical spine after C1-C2 transarticular screw fixation in rheumatoid arthritis. J Spinal Disord Tech. 2007;20(6):436-41.

28. Lapsiwala SB, Anderson PA, Oza A, Resnick DK. Biomechanical comparison of four $\mathrm{C} 1$ to $\mathrm{C} 2$ rigid fixative techniques: anterior transarticular, posterior transarticular, $\mathrm{C} 1$ to C2 pedicle, and C1 to C2 intralaminar screws. Neurosurgery. 2006;58(3):516-21. 